\title{
Turismo en Vilcabamba: Impacto y evolución del ingreso de turistas frente a la oferta turística del sector entre los años 2017 y 2018.
}

Tourism in Vilcabamba:

Impact and evolution of the income of tourists against the tourist offer of the sector between the years 2017 and 2018.

Ing. Gustavo Vladimir Paladines Mgtr. ${ }^{1}$, Ing. Jenny Elizabeth Suárez Velasco Mgtr. ${ }^{2}$, Dr. C. Eros Salinas Chávez. ${ }^{3}$ \& Dra. C. Maité Echarri Chávez. ${ }^{4}$

\begin{abstract}
.
DOI: https://doi.org/10.33262/cienciadigital.v9i2.377

Tourism in Vilcabamba has become an activity with high potential for the development of the town. Although this was initiated by the proliferation of rumors that the sector promotes good health and a long life, the network of forms of tourism that have impacted on the environment, customs and lifestyle has also been generated. population, such as residential tourism; or for the tourist offer managed by the private company and included by resident foreign tourists. For the development of the study, a descriptive research was carried out, a qualitative and quantitative approach, the application of population surveys, tourists, and interviews with representatives of the tourism sector of Vilcabamba. Among the results it is considered that the percentage of the population dedicated to tourism is one third of the total population, and a quarter of it rates tourism as negative due to its impact on the environment and the typical tranquility of Vilcabamba. It has also been shown that there is a gap between the entry of tourists and the tourist offer, which is lower than the proportion of visitors, which creates an opportunity to boost community tourism.
\end{abstract}

Keywords: Tourism, Residential Tourism, Vilcabamba.

\footnotetext{
${ }^{1}$ Universidad de las Fuerzas Armadas, Sangolquí, Ecuador, gvpaladines@ espe.edu.ec

${ }^{2}$ Universidad de las Fuerzas Armadas, Sangolquí, Ecuador, jesuarez1@espe.edu.ec

${ }^{3}$ Universidad de La Habana, Cuba, esalinas@ ftur.uh.cu

${ }^{4}$ Universidad de La Habana, Cuba, mecharri@ftur.uh.cu
} 


\section{Resumen.}

El turismo en Vilcabamba se ha convertido en una actividad con alto potencial para el desarrollo de la localidad. Si bien esto inició por la proliferación de rumores de que el sector promueve la buena salud y una larga vida, también ha generado el incremento de formas de turismo que han impactado en el medio ambiente, las costumbres y el estilo de vida de la población, como el turismo residencial; o por la oferta turística gestionada por la empresa privada e inclusive por turistas extranjeros residentes. Para el desarrollo del estudio se llevó a cabo una investigación descriptiva, con enfoque cualitativo y cuantitativo, mediante la aplicación de encuestas a la población, a los turistas, y entrevistas a representantes del sector turístico de Vilcabamba. Entre los resultados se observó que el porcentaje de población dedicado al turismo es cercano a la tercera parte de la población total, y que una cuarta parte de la misma califica al turismo como negativo debido a su impacto en el medio ambiente y la tranquilidad típica de Vilcabamba. Se evidenció también que existe una brecha entre el ingreso de turistas y la oferta turística, siendo esta menor a la proporción de visitantes, lo que crea una oportunidad para impulsar el turismo comunitario.

Palabras claves: Turismo, Turismo Residencial, Vilcabamba.

\section{Introducción.}

Entre los sectores económicos, el turismo destaca como una de las actividades productivas más diversas y amplias, pues su ejecución requiere de toda una cadena de servicios y productos que tienen como finalidad, mejorar la experiencia que vive el turista.

Vilcabamba es un valle ubicado a 40 kilómetros de Loja, capital de la provincia de Loja en Ecuador. Cuenta con un clima subtropical-seco, una temperatura media aproximada de $20,3^{\circ} \mathrm{C}$ y contiene cerca de 5000 habitantes (Municipio de Loja, 2018). Vilcabamba, conocido como el valle de la longevidad, tiene una reputación particular en el ámbito turístico. Este reconocimiento lo ganó a través de un estudio realizado por National Geographic en el año de 1973 en el que se hacía mención de pobladores de Vilcabamba que habían llegado a más de 120 años de vida (Rachowiecki \& Thur, 2008, pág. 248), sin embargo estudios posteriores no han confirmado esta información (Mazess \& Forman, 1979). Esta notoriedad atrajo la atención de viajeros de todo el mundo dando paso al llamado turismo de salud y, en la actualidad, el sector es valorado también por sus atractivos naturales, clima y la tranquilidad que reina en su ambiente.

No obstante, las oportunidades de trabajo e ingresos que ha generado el turismo en Vilcabamba, se ven opacadas por la afectación que puede producir al entorno medioambiental y a los estilos de vida, costumbres y tradiciones. En entornos donde los recursos naturales son el principal atractivo para el visitante, la sustentabilidad debe ser un factor clave. El turismo sustentable demanda alternativas en las que el impacto es controlado, 
y los ingresos provenientes del turismo permiten la sustentación de la población del sector, la protección de los atractivos naturales, y el cuidado de las tradiciones y elementos culturales que los identifica.

Por estas razones el turismo no solo debe ser sustentable, sino también rentable como actividad productiva. En este ámbito encaja la recreación como parte del turismo pues involucra actividades de ocio y relajación a las que el visitante puede acceder como parte de su estancia; sin embargo, esta definición es muy amplia y puede abarcar a varios tipos de turismo, por lo cual en el presente artículo se diferenciará del turismo tradicional con fines recreativos (comprendiendo a todas las actividades turísticas que promueven la relajación, esparcimiento y/o diversión) y el turismo no tradicional (como el turismo de salud y el turismo residencial, ambos con presencia en Vilcabamba).

Vilcabamba es, en la actualidad, un destino turístico que ofrece, a más de los atractivos naturales y culturales, un turismo de salud y residencial que no se ven con facilidad en otras ciudades del país.

En Vilcabamba el aire es ideal; ni muy cálido ni muy frío, con un fresco aroma de montaña y un toque de incienso en sus efímeras laderas. Esto confiere a la región un halo místico, irresistible para muchos. Quizá este sea el motivo por el cual en el lugar haya más negocios de extranjeros que en cualquier otro sitio de Ecuador. (St. Louis, Grosberg, Benchwick, \& Waterson, 2016)

El turismo residencial puede comprenderse como un fenómeno caracterizado por el "comportamiento móvil oscilante entre la migración permanente, la temporal y la mera movilidad” (Mantecón, 2008, pág. 108), con frecuencia realizado por jubilados o personas mayores según el mismo autor. El turismo de salud comprende a todas las alternativas que los turistas buscan y acceden para mejorar su salud, influenciados por la reputación del sector, de ser una comunidad centenaria.

Estos aspectos particulares del sector, han provocado una brecha entre el ingreso de turistas y la oferta turística recreativa que debería existir para satisfacer esta demanda. Un enfoque de la oferta direccionado a cubrir las necesidades y la demanda turística tendría un impacto económico más beneficioso y sustentable para la población que vive de los ingresos por turismo, pero debe acompañarse de políticas de protección, mantenimiento de los recursos naturales, y valoración del patrimonio cultural-social.

\section{Materiales y Métodos}

La investigación se basó en un diseño con enfoque mixto, al tratar los datos de manera cualitativa y cuantitativa.

El tipo de investigación aplicado se califica como descriptiva, ya que se recopilaron datos para describir la realidad del turismo que tiene lugar en Vilcabamba, y como sugieren 
Vogeler y Hernández (2018, pág. 52) la investigación turística tiene como finalidad formular y responder preguntas que ayuden a generar información útil para que las empresas del sector turístico actúen mejor en el mercado, para que las administraciones territoriales mejoren la gestión y protección de los destinos turísticos, y para contribuir a la labor de otros investigadores. Los datos del estudio facilitarán conocer cómo se ha desarrollado la oferta turística en contraste con el ingreso de turistas, lo que permitiría direccionar con mayor precisión los esfuerzos que realice la oficina de turismo del sector.

El levantamiento de encuestas se sustentó en una muestra poblacional aleatoria, con el 5\% de error de muestreo y una confianza del 95\%, en una población compuesta por 5000 habitantes (siendo este el valor aproximado de población que el Municipio de Loja registra para el año 2018). La muestra calculada fue de 357 personas.

$$
n=\frac{N \times z^{2} \times p \times q}{e^{2} \times(N-1)+z^{2} \times p \times q}=\frac{5000 \times 1,96^{2} \times 0,5 \times 0,5}{0,05^{2} \times(5000-1)+1,96^{2} \times 0,5 \times 0,5}=356,8 \approx 356
$$

Donde $\mathrm{N}=$ población, $\mathrm{z}=$ valor $\mathrm{z}$ para nivel de confianza, e= error de muestreo, $\mathrm{p}=$ probabilidad estándar e ocurrencia, q= probabilidad estándar de no ocurrencia

De manera adicional se entrevistó a tres representantes del ámbito turístico del sector, y se obtuvo información estadística de turismo otorgada por la Oficina de Turismo de Vilcabamba.

Como instrumento de investigación se utilizaron cuestionarios de encuesta a pobladores y turistas y una guía de entrevista a representantes del sector turístico. Los objetivos del estudio fueron:

- Determinar la percepción de la población acerca del turismo y su impacto.

- Establecer la percepción del turista sobre la oferta de servicios turísticos y su motivo de visita a Vilcabamba.

- Identificar una brecha entre la demanda turística y la oferta de turismo en Vilcabamba

\section{Resultados.}

Mediante la aplicación de las encuestas a pobladores del sector se pudo realizar una aproximación al panorama turístico de Vilcabamba desde la perspectiva de la población y de los turistas.

Como muestra el gráfico 1, la mayor parte de la población encuestada se encontró entre los 19 y 25 años, y de forma equivalente, entre las edades de entre 26 a 55 años. En cuanto al sexo la proporción de encuestados se reparte en un $52 \%$ de sexo masculino y un $48 \%$ de sexo femenino. 
Gráfico 1. Edad y sexo de los encuestados

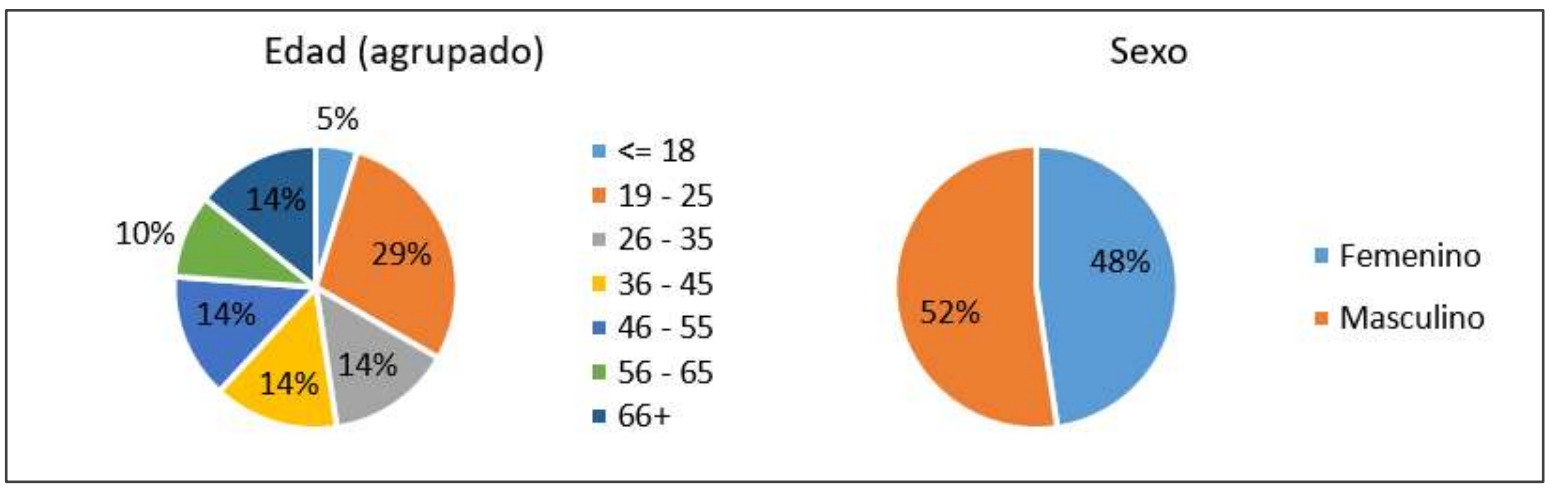

Fuente: Encuesta a pobladores de Vilcabamba

Respecto a la percepción sobre el impacto del turismo, el $71 \%$ de encuestados opina que se trata de algo positivo, frente a un $29 \%$ que lo califica como algo negativo según refleja la figura 2. Entre los encuestados que lo califican como positivo las opiniones se agrupan en que se promueve la generación de trabajo, el comercio y, por ende, los ingresos para la población, aunque solo el 28\% señala tener una ocupación que está relacionada con él turismo. Frente a esto, las opiniones sobre el turismo como algo negativo en el valle de Vilcabamba, expresan que el impacto medioambiental es fuerte, que se altera la tranquilidad en el estilo de vida, y se ven influenciadas las costumbres tradicionales.

Cabe resaltar aquí, que según Toledo (2018), representante de la Oficina de Turismo de Vilcabamba, los turistas que llegan a Vilcabamba buscan tranquilidad y en la actualidad visitan el sector pero no desean realizar exagerados gastos en servicios y planta turística, por lo cual en su mayoría buscan gratuidades, voluntariados e intercambios; lo que reduce los beneficios económicos que recibiría la localidad.

Gráfico 2. Percepción del turismo y ocupación relacionada con el turismo en Vilcabamba

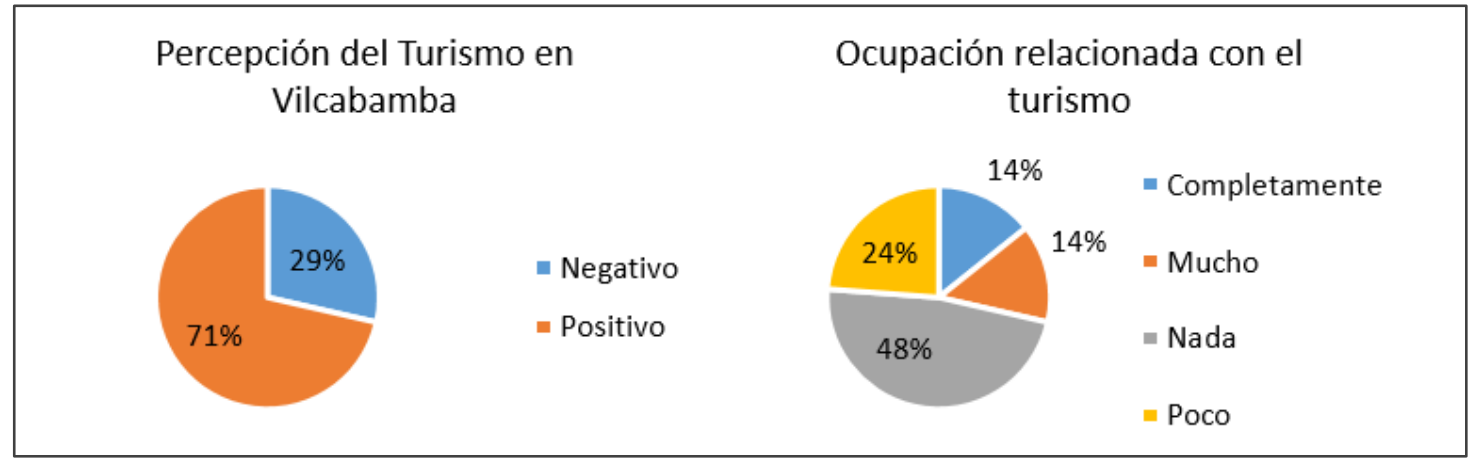

Fuente: Encuesta a pobladores de Vilcabamba 
La población que tiene una ocupación relacionada con el sector turístico se reparte en, un $40 \%$ en el ámbito del turismo de recreación, y un 30\% en alimentación, dejando un $10 \%$ en alojamiento como indica el gráfico 3, y porcentajes iguales en turismo de salud y artesanías; no obstante, estos porcentajes expresan la distribución de los encuestados ocupados en el sector turístico, la cual puede diferenciarse de la distribución total del sector. En todo caso, muestra una proporción mayor encaminada a recreación y alimentación.

Al indagar sobre la opinión respecto a la pérdida de costumbres, estilo de vida y prácticas tradicionales, el $66 \%$ se reparte entre de acuerdo y totalmente de acuerdo, lo que deja a un $34 \%$ con una opinión contraria. En conclusión, si bien solo una cuarta parte califica al turismo como algo negativo para el valle de Vilcabamba, más de la mitad es consciente del impacto en el estilo de vida.

Gráfico 3. Tipo de ocupación y opinión sobre la pérdida de costumbres, estilo de vida y tradiciones.

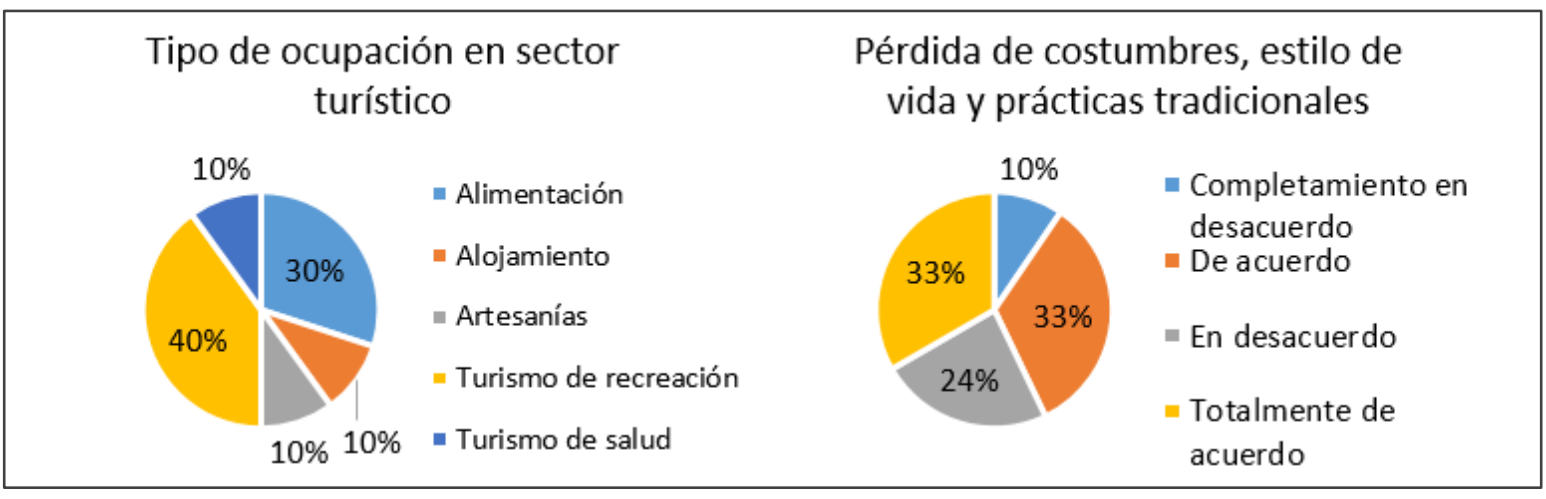

Fuente: Encuesta a pobladores de Vilcabamba

En este punto cabe resaltar la relevancia de este aspecto. De manera general las referencias que se realizan de Vilcabamba en la literatura [por ejemplo en Rudel (1992) y Ades y Graham (2003)], o en la promoción turística del sector, resaltan la tranquilidad en su estilo de vida y las costumbres tradicionales en cuanto a una alimentación sana basada en la agricultura y ganadería local, lo que de forma indirecta se relaciona con la creencia de una vida más larga en su población. Varios encuestados señalaron que, entre el impacto negativo, está la influencia mayoritaria en la población adolescente, en el ingreso desmedido de productos industrializados como bebidas gaseosas y otros manufacturados.

En la encuesta a turistas se tuvo, en su mayoría, a personas de 25 años o menos, siendo el $62 \%$ de los encuestados en este segmento como indica el gráfico 4.

Gráfico 4. Edad y aspectos que motivan a los turistas a visitar Vilcabamba 


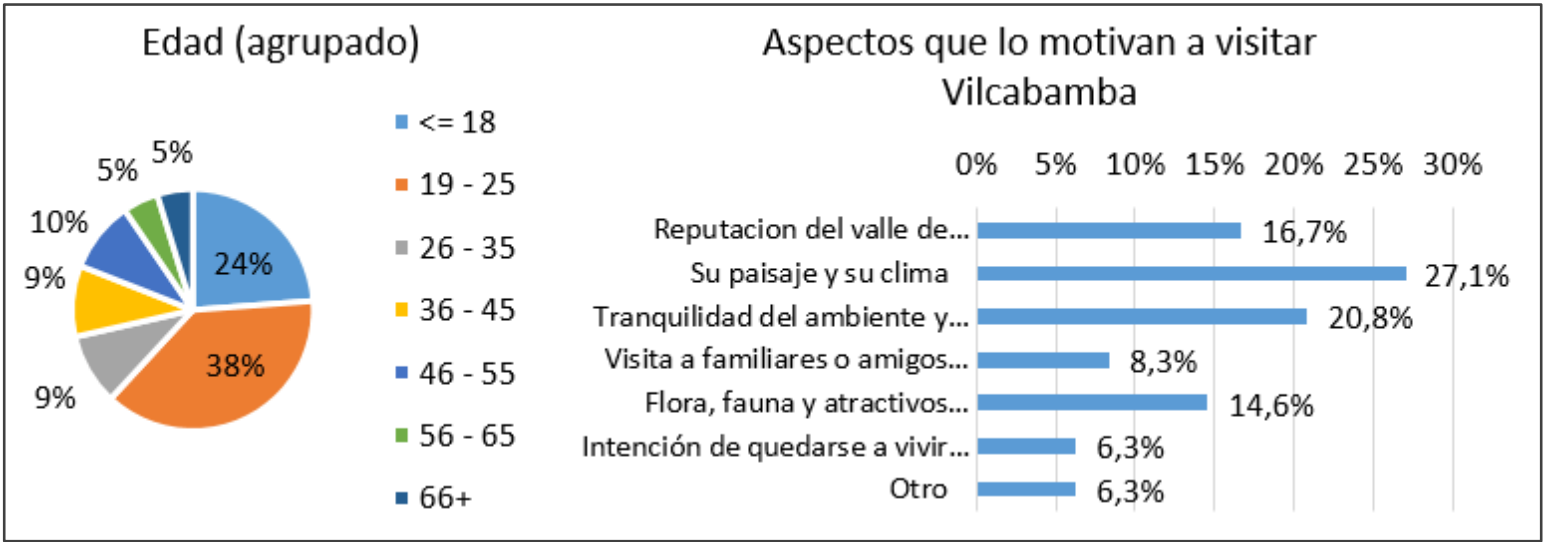

Fuente: Encuesta a pobladores de Vilcabamba

Los resultados permiten creer ver que existe una alta proporción de turistas jóvenes, no obstante, entre los encuestados se tuvo también sujetos de más de 65 años; sin embargo, los datos pueden no reflejar la distribución real de la población turística debido a que el turista joven suele encontrarse con mayor facilidad en los sitios de recreación por lo que se tuvo mayor facilidad de acceso a los mismos. Al indagar sobre las razones que motivaron al turista a visitar Vilcabamba, como indica el gráfico 4, la cuarta parte señaló que le atrajo su paisaje y su clima, seguido de una quinta parte que expresó la atracción por la tranquilidad del ambiente y el estilo de vida. El 16,7\% se refirió a su reputación como sitio que promueve la buena salud y una larga vida.

En el gráfico 5 se observa que el tiempo de estadía de la mayoría de turistas se encuentra entre 1 y 3 días, y entre 4 días y una semana, con un $43 \%$ y $29 \%$ respectivamente. La mayoría son turistas que visitan Vilcabamba de forma esporádica. Los datos también muestran que el $62 \%$ no posee familiares o amigos en Vilcabamba, no obstante, solamente el $14 \%$ se queda en casa de familiares o amigos.

Gráfico 4. Tiempo de estadía, familiares o amigos en Vilcabamba y sitio de hospedaje

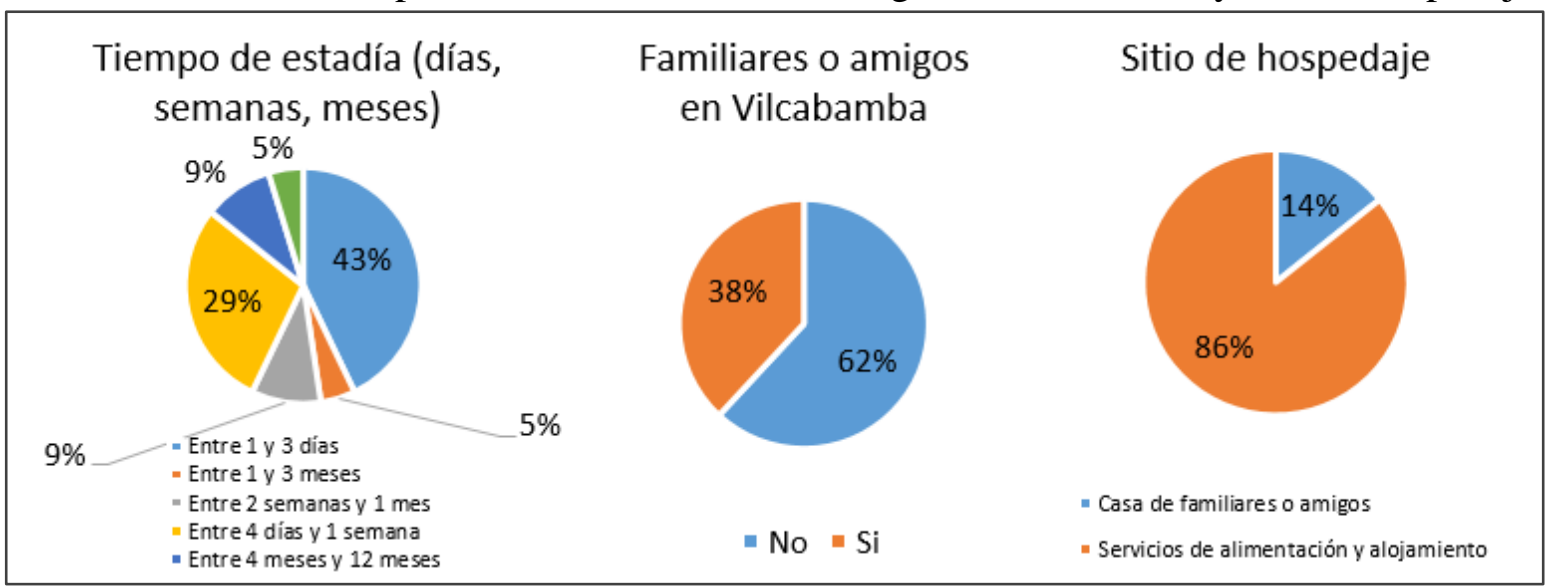


Fuente: Encuesta a pobladores de Vilcabamba

La información obtenida se complementó con los datos estadísticos recopilados. En el gráfico 6 se presentan los datos de turistas que ingresaron a Vilcabamba en el año 2017, clasificados por país, y en el segundo gráfico exceptuando a los turistas nacionales. En ese año ingresaron a Vilcabamba un total de 11726 turistas. El mayor porcentaje de turistas está compuesto por ecuatorianos, con un $63 \%$ del total; no obstante, al exceptuar a este segmento, se tiene que el $36 \%$ de turistas extranjeros son de Perú, seguidos por un 14\% de viajeros de Estados Unidos, Canadá con el 8\%, y Europa y Colombia con el $7 \%$ cada uno.

Gráfico 6. Estadísticas de ingresos de turistas a Vilcabamba, año 2017

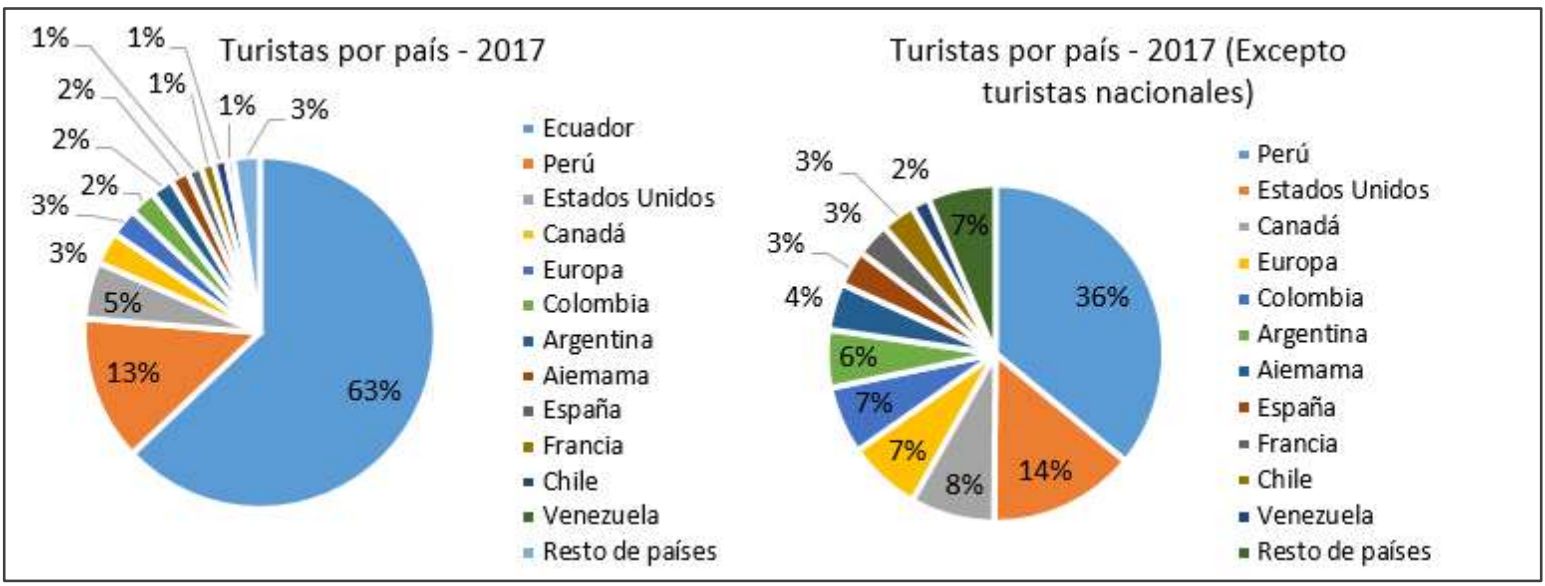

Fuente: (Oficina de Turismo de Vilcabamba, 2019), (Ministerio de Turismo, 2018)

En cuanto al año 2018, ingresaron 13219 turistas, lo que representa un crecimiento del 12,7\% en ese año. En este caso los turistas ecuatorianos representaron el $74 \%$ del total como muestra el gráfico 7. Al considerar solamente a extranjeros se obtuvo un $36 \%$ provenientes de Perú y el 12\% de Estados Unidos. Con un 7\% se encuentra España y con el 5\% tanto Francia como Colombia. Se mantiene constante el ingreso de turistas del Perú y de Estados Unidos como los grupos más numerosos de visitantes del extranjero.

Gráfico 7. Estadísticas de ingresos de turistas a Vilcabamba, año 2018 


\begin{tabular}{|l|lll|}
\hline $1 \%$ & Turistas por país - 2018 & & Turistas por país - 2017 (excepto \\
turistas nacionales)
\end{tabular}

Fuente: (Oficina de Turismo de Vilcabamba, 2019), (Ministerio de Turismo, 2018)

En el gráfico 8 se presenta la estacionalidad de la demanda turística comparada entre 2017 y 2018. En ambos casos los mayores flujos de turistas tienen lugar entre los meses de julio, agosto y noviembre, mientras que los más bajos corresponden a los primeros cinco meses, junto con una caída producida en septiembre y octubre.

Gráfico 8. Estacionalidad de la demanda turística años 2017 y 2018

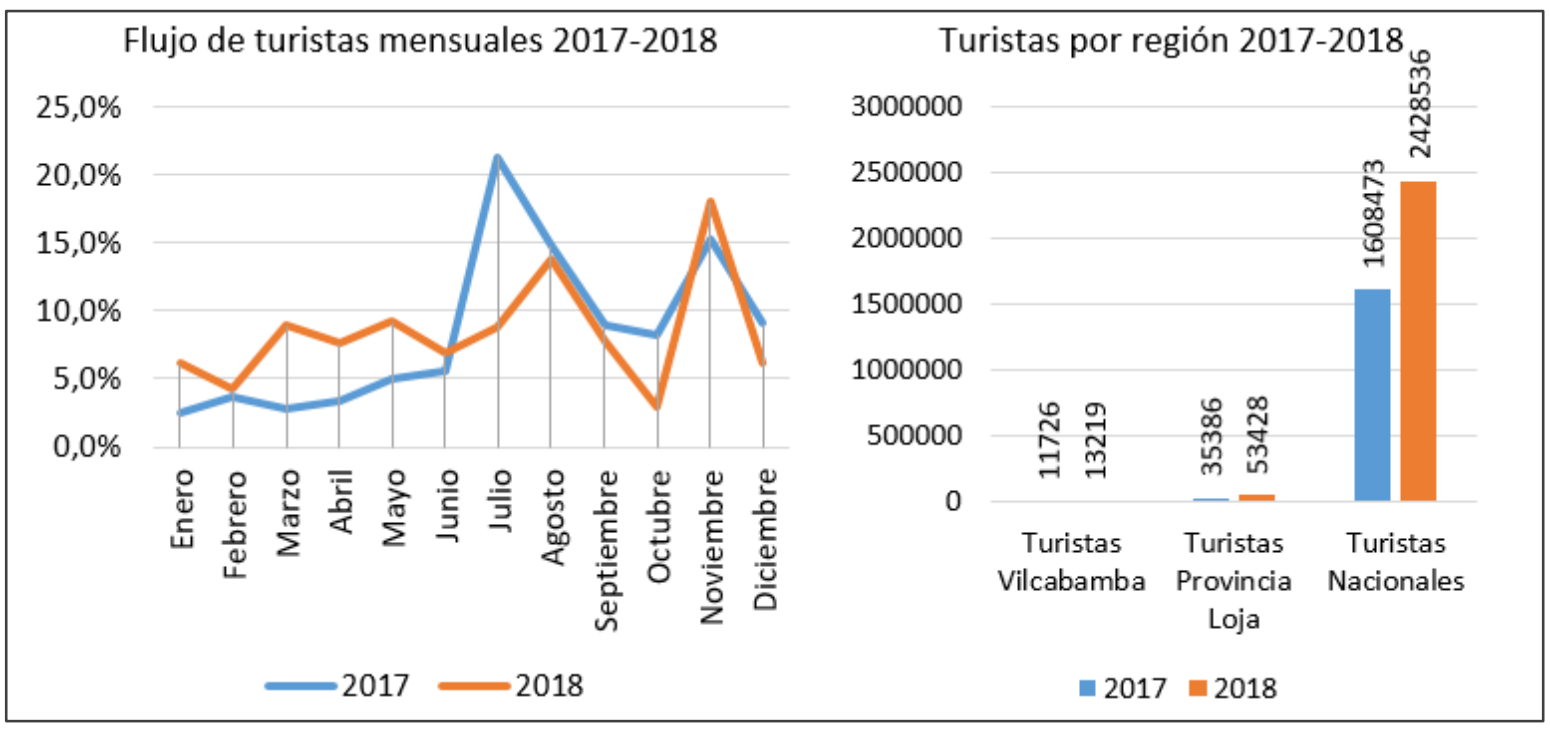

Fuente: (Oficina de Turismo de Vilcabamba, 2019), (Ministerio de Turismo, 2018).

En la tabla 1 se presentan los datos del total de turistas a nivel nacional, provincial y del sector de Vilcabamba. Entre el año 2017 y 2018 se produce un aumento del 50,98\% de turistas, pasando de 1'608473 visitantes del extranjero en el año 2017 a 2'428536 en el año 2018, para Loja esto significó un aumento anual de turistas del 44,1\%, sin embargo, para Vilcabamba el crecimiento fue solamente del $12,7 \%$. 
Tabla 1. Datos de turistas a nivel de población, provincia y nacional

\begin{tabular}{|c|c|c|c|}
\hline & Turistas Vilcabamba & Turistas Provincia Loja & Turistas Nacionales \\
\hline \multirow{4}{*}{2017} & 11726 & 35386 & 1608473 \\
\cline { 2 - 4 } & $33 \%$ del total provincial & $2.2 \%$ del total nacional & $100 \%$ \\
\cline { 2 - 4 } & $0,73 \%$ del total nacional & & \\
\hline \multirow{4}{*}{$\mathbf{2 0 1 8}$} & 13219 & 53428 & 2428536 \\
\cline { 2 - 4 } & $25,9 \%$ del total provincial & $2.1 \%$ del total nacional & $100 \%$ \\
\cline { 2 - 4 } & $0,54 \%$ del total nacional & & \\
\hline
\end{tabular}

Fuente: (Oficina de Turismo de Vilcabamba, 2019), (Ministerio de Turismo, 2019), (Valverde, 2018)

El turismo que tiene lugar en la provincia de Loja comprende cerca del $2 \%$ del turismo total nacional, mientras que Vilcabamba apenas suma el 0,73\% para el año 2017 y 0,54\% para el 2018 a nivel nacional. Frente al turismo de la provincia de Loja Vilcabamba representó el $33 \%$ y el $25,9 \%$ entre los años 2017 y 2018.

En la tabla 2 se presenta el promedio mensual de turistas en la provincia de Loja, en el cantón y en Vilcabamba, frente a la cantidad de establecimientos de alojamiento y de alimentos y bebidas registrados, así como las plazas disponibles en cada uno:

Tabla 2. Total, oferta de turismo en alojamiento y alimentos y bebidas, año 2018

\begin{tabular}{|c|c|c|c|c|c|}
\hline & \multicolumn{2}{|c|}{ Alojamiento } & \multicolumn{2}{c|}{ Alimentos y bebidas } \\
\cline { 2 - 6 } & $\begin{array}{c}\text { Turistas } \\
\text { mensuales } \\
\text { (promedio)- } \\
\text { (2018 }\end{array}$ & $\begin{array}{c}\text { Servicios de } \\
\text { alojamiento } \\
\text { registrados }\end{array}$ & $\begin{array}{c}\text { Plazas } \\
\text { disponibles }\end{array}$ & $\begin{array}{c}\text { Servicios de } \\
\text { alimentación } \\
\text { registrados }\end{array}$ & $\begin{array}{c}\text { Plazas } \\
\text { disponibles }\end{array}$ \\
\hline Provincia Loja & $\mathbf{4 2 5 0}$ & $\mathbf{1 1 3}$ & $\mathbf{4 1 1 1}$ & $\mathbf{3 7 0}$ & $\mathbf{1 7 8 4 6}$ \\
\hline Cantón Loja & 2996 & 63 & 2898 & 284 & 13716 \\
\hline $\begin{array}{c}\text { Vilcabamba (incluye no } \\
\text { catastrados) }\end{array}$ & 1102 & 25 & 699 & 52 & 1147 \\
\hline
\end{tabular}

Fuente: (Oficina de Turismo de Vilcabamba, 2019), (Ministerio de Turismo, 2019), (Valverde, 2018)

Los datos muestran que, para un promedio mensual de 1102 turistas en Vilcabamba, se tiene un total de 699 plazas disponibles en servicios de alojamiento, y 1147 plazas en servicios de alimentación y bebidas. Hay que considerar que estos datos se encuentran tanto los servicios catastrados como aquellos que aún no lo están.

En el caso de servicios de turismo en salud solo se tiene conocimiento del Centro de Meditación como local catastrado, no obstante una representante de la Administración Distrital de Vilcabamba (Ambuludi, 2018) señaló que existen cerca de 20 sitios que ofrecen 
servicios de spa, tratamientos rejuvenecedores, masajes; mientras que una operadora turística (Carpio, 2018) mencionó también servicios de yoga, servicios de spa en hosterías, e inclusive sesiones espiritistas, aunque estas no forman parte de la oferta turística.

\section{Discusión}

Los datos recopilados muestran que el turismo en Vilcabamba es una actividad con fuerte potencial pero que aún no se ha desarrollado de manera eficiente. Por un lado, las plazas disponibles son bajas en comparación al total de plazas de la provincia con 699 plazas en servicios de alojamiento frente a 4111 lo que representa el 17\%, mientras que el promedio de clientes de Vilcabamba frente al promedio de clientes en la provincia es del 26\%; es decir, la proporción de plazas disponibles de Vilcabamba frente a toda la provincia es mucho menor a la proporción de turistas que atrae. En el caso de servicios de alojamiento es menor aún, con un $6,4 \%$ del total de plazas disponibles provinciales.

El desarrollo turístico en Vilcabamba tampoco puede calificarse como un proceso sustentable, pues un gran número de los servicios dedicados al turismo son empresas privadas o servicios que pertenecen a extranjeros que optaron por un turismo residencial, de forma que no todos los residentes, o al menos la gran mayoría, se ha visto beneficiados con el turismo. Al respecto cabe señalar lo dicho por Nel-lo, Campos y Sosa (2015), para quienes el turismo residencial, aparte de constituir un dilema conceptual por tratarse de opuestos (turista y residente como antónimos), es un fenómeno complejo por el impacto que genera en el territorio, principalmente por cambios "en la organización y uso del territorio, creación, reformulación, rearticulación y desarticulación de espacios en las economías de los destinos y en las comunidades receptoras" (Nel-lo, Campos, \& Sosa, 2015, pág. 440).

Por otro lado, Mazón (2018) señala que el segmento compuesto por turistas residentes influye en una mayor demanda de turismo de salud, pues:

Como consecuencia del aumento del tiempo libre y de ocio, así como de las nuevas pautas de consumo y bienestar personal, aumenta la búsqueda de una mejor calidad de vida, lo que conlleva también una demanda de mayor atención a la salud y al cuidado personal. (Huete, 2007 citado en Mazón, 2018, pág. 136)

En este sentido uno de los factores que influyen en el aumento del turismo de salud en Vilcabamba, es que es un lugar que reúne las características buscadas por turistas residenciales, pues "el hecho de permanecer largos períodos de tiempo alejados de su país de origen, así como la media de edad de este tipo de población, sí los convierte en usuarios de los servicios de salud de los países donde se ubican” (Mazón, 2018, pág. 136). El turista residencial generalmente es un extranjero o parejas de extranjeros, jubilados, con gran tiempo libre, y ahorros suficientes para la adquisición de bienes inmuebles. No obstante, el aumento en el turismo residencial implica que muchos de estos turistas inicien también servicios 
turísticos privados en los que la comunidad participa poco o de forma indirecta, de forma que los beneficios del turismo no se direccionan principalmente a la sustentabilidad del entorno.

La reputación obtenida por Vilcabamba a nivel mundial como un sector que reúne las características para vivir con buena salud y alcanzar edades muy avanzadas, fueron un primer detonante para atraer el turismo residencial, no obstante, estudios posteriores no han comprobado la información señala por National Geographic en la década de los Setenta, como sugiere Mazess y Mathisen (1982). Sin embargo, en la actualidad los cambios acaecidos en los hábitos y estilos de vida, y de alimentación, pueden ser también una razón de que no se puedan encontrar hallazgos con la misma facilidad; o bien puede existir una ausencia de estudios longitudinales que demuestren o refuten en la población del sector (incluyendo a pobladores agrarios ubicados en áreas alejadas del centro urbano de Vilcabamba) el alcance de edades centenarias.

Para Gascón (2016) el turismo residencial también trae consigo problemas adicionales. Con el aumento del turismo los ingresos aumentan sin embargo los precios de los productos agrícolas no lo han hecho a la par, lo que ha incidido en que, con el paso del tiempo, la fuerza de trabajo agrícola se haya reducido, modificándose inclusive con esto el tipo de agricultura realizada. Se han reducido los cultivos de ciclo corto que requieren mayor fuerza de trabajo (como hortalizas y cereales) y se ha dado paso a cultivos de ciclo largo como caña o frutos leñosos. A su vez esto impacta en la modificación de los hábitos de consumo y dieta de la población local.

La brecha existente entre servicios de turismo y recreación, y la cantidad de turistas que arriban a Vilcabamba es también producto de la falta de visión y aprovechamiento del turismo como actividad productiva por parte de la población local. Hayes (2015) califica los proyectos de vida de los pobladores como individualistas y utilitaristas, manteniendo relaciones sociales de subordinación; y no emprendiendo proyectos comunitarios ni sociales en ningún sentido. Es notorio también el propio estilo de vida tradicional de los pobladores, que anhelan la tranquilidad y la salud, y que ha influido en no emprender proyectos turísticos comunitarios, dejando en el sector privado la responsabilidad y las oportunidades que el turismo conlleva.

\section{Conclusiones.}

- El turismo en Vilcabamba se caracteriza por servicios esporádicos dedicados a la recreación, y una oferta mayor en servicios de alojamiento y alimentación; si bien el desarrollo de estos es menor al volumen de ingreso de turistas, lo que abre la oportunidad a la implementación de nuevos proyectos que complementen la oferta turística del sector. 
- Sin embargo, el sector ofertante de turismo en Vilcabamba está compuesto por servicios administrados de forma privada, y más aún, un gran número de negocios más pequeños administrados por turistas extranjeros residentes.

- El turismo no es algo negativo, aunque así lo perciba parte de su población, sin embargo, esto refleja insatisfacción en este colectivo respecto a cómo se está desarrollando el turismo en el sector. A pesar del potencial turístico del sector, la proporción de pobladores que participan en ámbito es bajo, por lo cual no todos reciben un beneficio directo del mismo, lo que reduce su potencial de ser una actividad sustentable para la población en general. Más aún, existe un gran número de locales o servicios turísticos gestionados y pertenecientes a extranjeros, lo que ha desplazado las posibilidades de la propia población. El crecimiento de una actividad productiva como el turismo siempre es positivo, y si bien esto tiene un impacto directo en múltiples facetas, esto puede ser controlado, con mayor razón si el mantenimiento de las tradiciones y estilo de vida apoya la generación de un potencial para el turismo cultural.

\section{Recomendaciones}

Vilcabamba ha ganado reconocimiento mundial por su calificativo de valle de la longevidad, y más allá de si esto es verídico o no, esto se ha convertido en un fuerte factor potencial para atraer el turismo, aunque la población local no ha trabajado en comunidad para aprovechar esta situación. Se recomienda a las administraciones municipales promover iniciativas que permitan desarrollar el turismo comunitario y sostenible, para integrar a la propia comunidad en este ámbito, y de esta forma fortalecer la valoración de las propias costumbres y tradiciones como una fortaleza turística.

Es también necesario fortalecer el marco regulador territorial, para controlar el impacto que el turismo residencial puede provocar, de forma principal en el medio ambiente. El incremento en este tipo de turismo atrae también a inversión para proyectos inmobiliarios, lo que puede alterar completamente y debilitar el estilo de vida y ambiente de tranquilidad que caracteriza en la actualidad a Vilcabamba.

Por último, sería recomendable la implementación, por parte de las entidades encargadas del turismo en el sector, el impulsar programas de información, concienciación y capacitación en temas turísticos, para promover otras formas de turismo como el gastronómico, patrimonial, comunitario, entre otros; y así lograr un desarrollo más controlado, en el que los ingresos por turismo se dirijan a la propia población y al cuidado del valle de Vilcabamba. 


\section{Referencias bibliográficas.}

Ades, H., \& Graham, M. (2003). The Rough Guide to Ecuador. New York, USA: Rouch Guides.

Ambuludi, A. (18 de Febrero de 2018). Entrevista. (G. Paladines, Entrevistador)

Carpio, M. (13 de Febrero de 2018). Entrevista. (G. Paladines, Entrevistador)

Gascón, J. (2016). Turismo residencial y crisis de la agricultura campesina. Los casos de Vilcabamba y Cotacachi (Andes ecuatorianos). Revista de Turismo y Patrimonio Cultural, 14(2), 309-318.

Gutiérrez, F. (2015). Apuntes de conceptos básicos para muestreo estadístico. Guadalajara, México: Lulu Press, Inc.

Hayes, M. (2015). Into the Universe of the Hacienda: Lifestyle Migration, Individualism and Social Dislocation in Vilcabamba, Ecuador. Journal of Latin American Geography, 14(1), 79-100.

Mantecón, A. (2008). La experiencia del turismo: un estudio sociológico sobre el proceso turístico- residencial. Barcelona, España: Icaria Editorial.

Mazess, R., \& Forman, S. (1979). Longevity and Age Exaggeration in Vilcabamba, Ecuador. Journal of Gerontology, 34 (1), 94-98.

Mazess, R., \& Mathisen, R. (1982). Lack of Unusual Longevity in Vilcabamba, Ecuador. Revista Human Biology, 54(3), 517-524.

Mazón, T. (2018). Turismo residencial: Nuevos estilos de vida: De turistas a residentes. España: Universidad de Alicante.

Ministerio de Turismo. (2018). Perfil de Turismo Internacional . Obtenido de Ministerio de Turismo: $\quad$ http://servicios.turismo.gob.ec/descargas/Turismocifras/Publicaciones/Perfiles/PerfilesDeTurismoInternacional.pdf

Ministerio de Turismo. (2019). Establecimientos Registrados. Obtenido de Ministerio de Turismo: http://servicios.turismo.gob.ec/index.php/turismo-cifras/2018-09-18-2111-17/establecimientos-registrados

Municipio de Loja. (2018). Vilcabamba: Datos Generales y Geográficos. Obtenido de Municipio de Loja: http://www.loja.gob.ec/contenido/vilcabamba

Nel-lo, A., Campos, B., \& Sosa, A. (2015). Temas pendientes y nuevas oportunidades en Turismo y Cooperación al Desarrollo. Coodtur. 
Oficina de Turismo de Vilcabamba. (2019). Estadísticas de turismo. Investigación propia.

Rachowiecki, R., \& Thur, M. (2008). Ecuador: Climbing and Hiking Guide. Viva Publishing Network.

Rudel, C. (1992). L' Equateur. Paris, Francia: KARTHALA Edition.

St. Louis, R., Grosberg, M., Benchwick, G., \& Waterson, L. (2016). Ecuador y las Islas Galápagos. España: Grupo Planeta Spain.

Toledo, D. (18 de Febrero de 2018). Entrevista. (G. Paladines, Entrevistador)

Valverde, L. (2018). Análisis de la oferta turística de la parroquia Vilcabamba, del cantón Loja. Trabajo de Titulación, Universidad Técnica Particular de Loja, Carrera de Administración de empresas turísticas y hoteleras, Loja, Ecuador.

Vogeler, C., \& Hernández, E. (2018). Introducción al Turismo: Análisis y estructura. Madrid, España: Editorial Universitaria Ramón Areces, S.A.

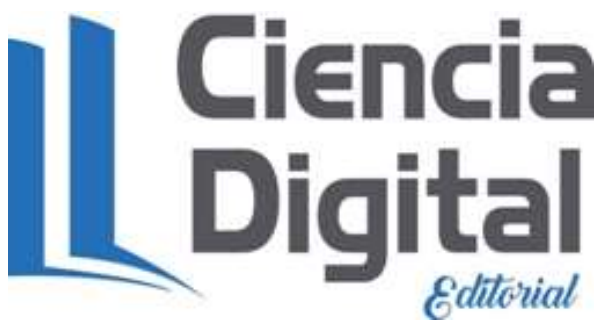




\section{Para citar el artículo indexado.}

Paladines G., Suárez J., Salinas E. \& Echarri M. (2019). Turismo en Vilcabamba: Impacto y evolución del ingreso de turistas frente a la oferta turística del sector entre los años 2017 y 2018. Revista electrónica Ciencia Digital 3(2), 93-108. Recuperado desde: http://cienciadigital.org/revistacienciadigital2/index.php/CienciaDigital/article/view/377/823

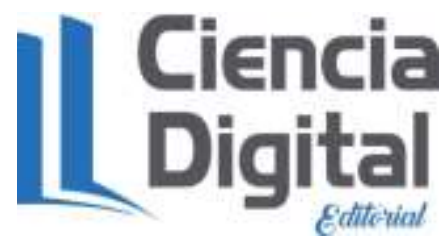

El artículo que se publica es de exclusiva responsabilidad de los autores y no necesariamente reflejan el pensamiento de la Revista Ciencia Digital.

El articulo queda en propiedad de la revista y, por tanto, su publicación parcial y/o total en otro medio tiene que ser autorizado por el director de la Revista Ciencia Digital.
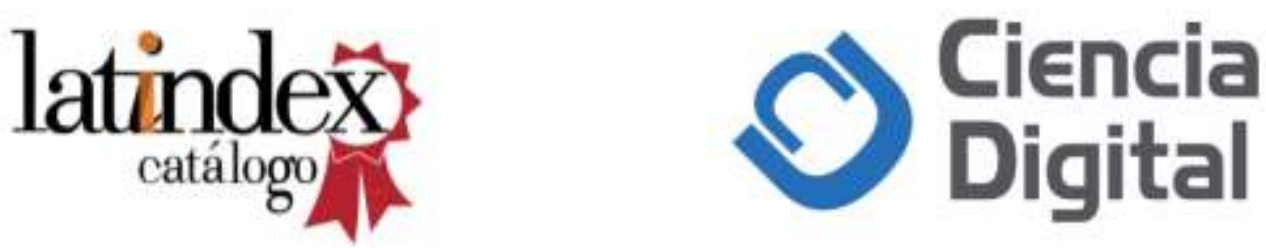